\title{
CoviD-19 Care of the dying person before and during the COVID-19 pandemic: A quality improvement project
}

\author{
Authors: Simon Tavabie, ${ }^{\mathrm{A}}$ Steve Bass, ${ }^{\mathrm{B}}$ Eleanor Stewart, ${ }^{\mathrm{C}}$ Ethan Redmore ${ }^{\mathrm{C}}$ and Ollie Minton ${ }^{\mathrm{D}}$
}

The current COVID-19 pandemic has necessitated the redeployment of NHS staff to acute-facing specialties, meaning that care of dying people is being provided by those who may not have much experience in this area. This report details how a plan, do, study, act (PDSA) approach was taken to implementing improved, standardised multidisciplinary documentation of individualised care and review for people who are in the last hours or days of life, both before and during the COVID-19 pandemic. The documentation and training produced is subject to ongoing review via the specialist palliative care team's continuously updated hospital deaths dashboard, which evaluates the care of patients who have died in the trust. We hope that sharing the experiences and outcomes of this process will help other trusts to develop their own pathways and improve the care of dying people through this difficult time and beyond.

KEYWORDS: COVID-19, end of life, dying, palliative

DOI: 10.7861/fhj.2020-0047

\section{Background}

Around half of all deaths in the UK occur in hospital, and the majority of people dying in this setting are cared for by clinical teams with experience in, but not specialism in, end-of-life care. ${ }^{1}$ Previous attempts to produce standardised guidance and pathways for the care of dying people have been mired in controversy. ${ }^{2}$ In response to concerns around individualising care, a report produced between multiple stakeholder organisations and in consultation with patient advocacy groups described our 'one chance to get it right', addressing the five priorities of care when it is thought a person may die within the next few days or hours: ${ }^{3}$

> This possibility is recognised and communicated clearly, decisions are made and actions taken in accordance with the

Authors: APalliative medicine registrar, Marie Curie Hospice, Hampstead, UK; Blead nurse for palliative and end-of-life care, Brighton and Sussex University Hospitals NHS Trust, Brighton, UK; ${ }^{C}$ core medical trainee, Brighton and Sussex University Hospitals NHS Trust, Brighton, UK; ${ }^{D}$ Macmillan clinical lead for palliative medicine, Brighton and Sussex University Hospitals NHS Trust, Brighton, UK person's needs and wishes, and these are regularly reviewed and decisions revised accordingly.

> Sensitive communication takes place between staff and the dying person, and those identified as important to them.

> The dying person, and those identified as important to them, are involved in decisions about treatment and care to the extent that the dying person wants.

$>$ The needs of families and others identified as important to the dying person are actively explored, respected and met as far as possible.

> An individual plan of care, which includes consideration of nutrition and hydration, symptom control and psychological, social and spiritual support, is agreed, co-ordinated and delivered with compassion.

Whether these five priorities are met is often used as a measure of the quality of care at the end of a person's life and is among the criteria used for the National Audit of Care at the End of Life, which informed the design of the representative "hospital deaths dashboard' process. ${ }^{4,5}$

Prior to the COVID-19 pandemic, plan, do, study, act (PDSA) quality improvement methodology was applied to the development of a new, multidisciplinary care plan with concurrent education sessions to improve the ability of clinical teams to provide good quality care at the end of life. ${ }^{6}$ During the COVID-19 pandemic this process was extended to support staff facing the new challenges that have arisen.

\section{Before the COVID-19 pandemic}

Delivering five priorities guided care can often be challenging for teams not familiar with this. ${ }^{7}$ Previous efforts had resulted in multiple fragmented and underutilised paper documents and areas of poor performance. It was initially acknowledged by the project team that a multidisciplinary care plan for people at the end of their lives would serve a multitude of purposes. For the clinicians, this would be as a structure for documenting symptom management plans (and accessing guidance for this) and recording conversations around goals of care and individual people's wishes in a format allowing reference by teams to ensure these needs were met. For nursing colleagues this would be a précis of the holistic daily care provided, symptom burden, and actions taken to ensure priorities were met.

Early PDSA cycles had involved focus groups with senior and junior doctors, nursing and care staff and senior trust managers from acute, general and specialty medical and surgical teams. 
Table 1. Case note reviews pre- and post-implementation of new individualised care plan for the dying person

$\begin{array}{lll}\text { Priority of care } & \text { Pre-implementation }(\mathbf{n}=\mathbf{4 0}) & \text { Post-implementation }(\mathbf{n}=\mathbf{8 0}) \\ \text { Recognition } & 80 \% & 89 \% \\ \text { Communication with dying person } & 31 \% & 46 \% \text { (where documented as impossible in further 35\%) } \\ \text { Communication with nominated person } & 91 \% & 91 \% \\ \text { Involvement in decision making } & 55 \% & 86 \% \\ \text { Needs of nominated person } & 33 \% & 58 \% \\ \text { Individualised plan of care } & 33 \% & 80 \%\end{array}$

These groups focused on what the healthcare professionals felt they needed support with, alongside their priorities for documenting plans and care provided. It was felt overwhelmingly that this care plan could provide less experienced staff with a guide to end-of-life care as well as helping them document their activity. Once collaboratively developed, an MDT care plan was piloted on four ward sites (oncology, complex discharge and care of the elderly wards) and direct feedback was collected from the nursing and medical staff. Together with case note review via the hospital deaths dashboard process, this feedback was used to inform multiple redesigns and eventual trust-wide roll out. ${ }^{4}$

Feedback from initial MDT focus groups of junior doctors $(n=11)$ suggested that that the care plan should be:

$>$ attractive ( $82 \%$ of respondents)

$>$ intuitive $(91 \%)$

$>$ brief $(73 \%)$

$>$ a précis of relevant information, not duplication (73\%)

$>$ inclusive of guidance on management $(100 \%)$.

Priorities for the project working group were that the final document would serve as a step-by-step guide for providing individualised care and as a way to capture of information for audit. Case note reviews were carried out with focus on whether there was evidence that the 'Five priorities of care' had been addressed in the person's end-of-life care (Table 1).

Overall care was rated as adequate or better in $84 \%$ of preintervention and $91 \%$ of post-intervention case note reviews. Areas of particularly poor practice, for example around hydration and nutrition, were identified and triggered educational intervention. There was an appreciation that case note review can only give a limited assessment of some aspects of end-of-life care and that a standardised document would have been helpful. Feedback collected during the last PDSA cycle was generally positive around the experience of using the document (Supplementary material: S1), but highlighted that the experience of accessing the document via the Trust's intranet often posed a barrier to its use.

\section{During the COVID-19 pandemic}

'I'm operating outside of my comfort zone and I'm always worried I'm not getting things right' - consultant physician

During the COVID-19 pandemic, large numbers of healthcare professionals have left their normal roles to join acute-facing specialties. As a result, end-of-life care is often being provided by those less familiar with the nuances of this, and specialist palliative care teams need to respond by adjusting the assistance they offer through review of their activity and resources. ${ }^{7.8}$ People less experienced or comfortable in delivering care for patients who are dying may find conversations about this more difficult and require support. ${ }^{1}$ Having earlier identified that document redesign, the provision of clear guidance and ongoing review of this could support staff in providing individualised care at the end of a person's life, a further PDSA cycle was commenced. Key identified objectives for this cycle were of improved visibility, accessibility and usability for a wider group of healthcare professionals.

\section{Plan}

To develop a care plan for the dying person that can be used in the context of the COVID-19 pandemic by adapting the current plan to staff and patient need.

\section{Do}

The guidelines for anticipatory prescribing in end-of-life care were updated to include specific guidance around management of symptoms in COVID-19 within the care plan (Supplementary material: S2). The documentation was updated and publicised via the Trust's Microguide ${ }^{T M}$ mobile and intranet apps ${ }^{9}$ alongside flashcards, posters and ward and team based educational intervention (Supplementary material: S3, S4).

\section{Study}

Initially, staff feedback was collected informally through discussions after educational events. It was felt that specific guidance around pharmacological and non-pharmacological management of symptoms in COVID-19 within the MicroGuide ${ }^{\mathrm{TM}}$ application and care plan itself was particularly useful for clinicians. Questionnaires were then circulated to junior doctors regarding the care plan.

\section{Results}

Discussions with nurses and doctors of varying grades on the wards elicited further useful feedback around their experiences in managing peoples' care at the end of their lives:

'I think the redeployed doctors and nurses have been reticent to give PRN medications and start syringe drivers.'

- core medical doctor.

'I've used the care plan to teach some of the junior doctors and nurses but it's tough when people aren't used to it.'

- medical ward nurse.

'Conversations around dying are really difficult when you're out of practice. Having practical guidance on what needs to be done is useful.

- senior medical doctor. 
Table 2. Provision of individualised end-of-life care 2 months preceding and during the COVID-19 pandemic

$\begin{array}{lll} & \text { Pre-pandemic data }(\mathbf{n}=\mathbf{2 4}) & \text { Pandemic data }(\mathbf{n = 1 5}) \\ \text { Average days to recognition of dying } & 8.8 & 8 \\ \text { Average days between recognition of dying and death } & 3.4 & 2 \\ \text { Evidence of treatment escalation plan } & 21 \% & 73 \% \\ \text { Evidence of communication with patient } & 58 \% & 60 \% \\ \text { Evidence of communication with next of kin } & 88 \% & 93 \% \\ \text { Evidence of involvement in planning } & 91 \% & 93 \% \\ \text { Evidence of support } & 79 \% & 80 \% \\ \text { Evidence of individualised care plan with review } & 69 \% & 73 \% \\ \text { Evidence of anticipatory prescribing } & 75 \% & 73 \% \\ \text { Evidence of discussion of hydration and nutrition } & 75 \% & 73 \% \\ \text { Subjective overall assessment of care adequate or better } & 92 \% & 93 \%\end{array}$

Generally, the feedback received commented on the improved usability and speed of update that digital resources offer. There was wider acknowledgement that care plans and guidelines form one part of the intervention and that ongoing educational support was needed to ensure practice change and improved comfort in managing patients at the end of their lives.

\section{Discussion}

Information gathered through the hospital deaths dashboard (Table 2) gives a snapshot of the care being delivered in the trust. ${ }^{4}$ While small variations are seen within each metric, there is no significant deterioration in the five priorities guiding end-of-life care provision. This is in spite of the involvement of healthcare professionals who are less familiar with this and a feeling that this might be affecting care delivery by the staff providing feedback earlier.

The average time between recognition of dying and patient death is shorter, which may be a result of the nature of COVID-19 deaths, or could suggest that their dying is identified later in the process. Indeed, though familiarity with caring for dying patients may be key to comfort in this recognition, experienced clinicians often perform poorly when trying to predict patient prognosis. ${ }^{8}$

A key area of improvement is in the use of clear treatment escalation planning to guide patient care when their clinical presentation changes. This is captured by the hospital deaths dashboard and continuing to promote this form of advance care planning may offer clinicians another prompt to consider that their patient may be dying in the face of any change in their clinical status. Given the comments discussed earlier, it is clear that though clinicans may feel 'out of practice' and 'reticent', they were able to discuss sensitive topics such as possible future deteriorations and prescribe anticipatory medications.

\section{Act}

Future PDSA cycles will focus on:

$>$ promoting the continued practice of treatment escalation planning and using this as a prompt to consider whether patients who are clinically deteriorating are dying
> ongoing data collection via the hospital deaths dashboard process to inform responsive redesign of documentation in light of new challenges ${ }^{4}$

> carrying out focus groups on a ward/team basis to understand barriers to provision of individualised end-of-life care where areas of poor practice are identified

> seeking feedback from local patient advocacy groups to ensure that the advocated 'five priorities of care' are sufficient for local need

$>$ exploring the experiences of clinical staff providing end-oflife care during the pandemic qualitatively, and probing the underlying philosophical, emotional and practical barriers that may be present.

\section{Conclusion}

As we enter the next phase of the COVID-19 pandemic, we are likely to require flexibility and responsiveness in the way we practice. Educational and supportive needs for healthcare professionals should be assessed and addressed by making processes clear, with well-defined guidance and access to help when needed.

The QI approach we have used to guide us through this project has been helpful in framing it as an iterative process. The most important learning points for the project team are that responsiveness and flexibility to service demands are aided by a PDSA approach and that junior-level involvement and consultation is the best way to shape lasting interventions. Case note review is an imperfect measure of delivered care as some aspects may be challenging to document and hard for reviewers to identify. We acknowledge the lack of patient advocacy and consultation through this process as a weakness and seek to engage with local Healthwatch organisations. ${ }^{10}$

As has been found previously, standardisation of care can be damaging for the individualised approach we espouse. Due to this, we have focused our efforts on creating a care plan that acts as a precis of care, a reminder of guidelines and an audit tool while prompting clinical teams to consider the priorities of care for the dying person. Guidelines and protocols are felt to be useful, especially for clinicians whose experience is limited, and this has been found to be particularly true during the COVID-19 
pandemic where clinicians are working outside of their normal clinical practice. It is important to note that these guidelines do not replace expert advice and support from specialists in palliative care should be sought whenever there is uncertainty.

\section{Supplementary material}

Additional supplementary material may be found in the online version of this article at www.rcpjournals.org/fhj:

S1 - Individualised care plan for the dying person documentation (non-COVID-19-specific)

S2 - COVID-19 care plan for the dying person documentation S3 - Flashcard/poster guidance for end-of-life care in COVID-19

S4 - Flashcard/poster guidance for communication in the COVID-19 pandemic

\section{References}

1 Royal College of Physicians. Talking about dying: How to begin honest conversations about what lies ahead. RCP, 2018. Available from www.rcplondon.ac.uk/projects/outputs/talking-about-dyinghow-begin-honest-conversations-about-what-lies-ahead [Accessed 16 May 2020].

2 Department of Health and Social Care. More care, less pathway: a review of the Liverpool Care Pathway. DHSC, 2013. Available from www.gov.uk/government/publications/review-of-liverpool-carepathway-for-dying-patients [Accessed 16 May 2020].

3 Leadership Alliance for the Care of Dying People. One chance to get it right. Available from https://assets.publishing.service. gov.uk/government/uploads/system/uploads/attachment_data/ file/323188/One_chance_to_get_it_right.pdf [Accessed 16 May 2020].

4 Minton O, Ede C, Bass S et al. Hospital deaths dashboard: care indicators. BMJ Support Palliat Care 2020, in press (doi: 10.1136/ bmjspcare-2020-002223).

5 NHS Improvement. Plan, do, study, act (PDSA) cycles and the model for improvement. Available from https://improvement.nhs.uk/ documents/2142/plan-do-study-act.pdf [Accessed 16 May 2020].

6 Tavabie S, Ball K, Carrigan R. Part 2: Perspectives on dying from COVID-19: The rising tide. Available from https://blogs.bmj.com/ spcare/2020/04/22/part-2-perspectives-on-dying-from-covid-19the-rising-tide/ [Accessed 16 May 2020].

7 White N, Reid F, Vickerstaff $V$ et al. Imminent death: clinician certainty and accuracy of prognostic predictions. BMJ Support Palliat Care 2019, in press (doi: 10.1136/bmjspcare-2018-001761).

8 Davies A, Hayes J. Palliative care in the context of a pandemic: similar but different. Clin Med 2020;20:274-7.

9 Brighton and Sussex University Hospitals NHS Trust. End of life and palliative care. Available from https://viewer.microguide. global/BSUH/COVIDBSUH\#content,61b0ca29-ecb1-45f0-9d15572ef80f78a3 [Accessed 16 May 2020].

10 National Association for Patient Participation. PPGs and Healthwatch. www.napp.org.uk/healthwatch.html [Accessed 16 May 2020].

Address for correspondence: Dr Simon Tavabie, Marie Curie Hospice, 11 Lyndhurst Gardens, Hampstead, London NW3 5NS, UK.

Email: simon.tavabie@nhs.net 